\title{
La articulación radiocubital distal. Anatomía, patología y tratamiento
}

\author{
L. A. Laurentin Pérez ${ }^{(1)}$, K. Ozer ${ }^{(2)}$, O. Díaz Casado ${ }^{(3)}$, L. R. Scheker ${ }^{(4)}$ \\ ${ }^{(1)}$ Senior Fellow Instituto Christine M. Kleinert de Cirugía de Mano y Microcirugía. \\ ${ }^{(2)}$ StafF Surgeon Department of Orthopedics, DenVer Health Medical Center, University of \\ COLORADO HEALTH SCIENCES CENTER. \\ ${ }^{(3)}$ Fellow Instituto Christine M. KLeinert de Cirugía de Mano y Microcirugía. \\ (4)Profesor de Cirugía Plástica, Cirugía de Mano y Microcirugía. Instituto Christine $M$. \\ KLeinert de Cirugía de Mano y Microcirugía Y UNIVERSidad de Louisville.
}

Correspondencia:

e-mail: lscheker@kleinertkultz.com

Rev. Iberam. Cir. Mano - Vol. 34 - Núm. 2 - noviembre 2006 (6-20)

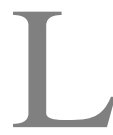

a rotación del antebrazo depende de una normal relación anatómica en las articulaciones radiocubital, distal y proximal, la membrana interósea, y la conformación anatómica de los huesos del antebrazo. Las lesiones de alguna de estas estructuras resulta en dolor, disminución de fuerza, limitación del movimiento y pérdida de la función del antebrazo.

\section{ANATOMÍA Y BIOMECÁNICA}

El radio y el cúbito constituyen una articulación bicondilar, articulados proximal y distalmente en las articulaciones radio-cubitales. Ubicada entre ambas articulaciones se encuentra la membrana interósea que actúa como ligamento suspensorio, dando origen a los músculos flexores y extensores. La estabilidad de las articulaciones radiocubital proximal y distal, depende de gruesas y complejas estructuras ligamentarias, conocidas como el ligamento anular y el complejo del fibrocartílago triangular (CFCT), respectivamente.

La articulación radio-cubital distal consiste en dos superficies articulares disímiles, la escotadura sigmoidea y la cabeza del cúbito. El contacto de estas dos superficies articulares varía del $80 \%$ de contacto, durante la posición neutra, al $10 \%$ o $20 \%$ durante la pronación y supinación máximas ${ }^{1}$. La articulación se en- cuentra estabilizada, fundamentalmente, por el $\mathrm{CFCT}^{1}$, el cual consiste en el fibrocartílago triangular (disco articular) ${ }^{2}$, los ligamentos radiocubital palmar y dorsal ${ }^{3}$, el menisco homó$\log _{0} 4$, y la vaina del cubital posterior ${ }^{2}$. Los ligamentos radiocubital palmar y dorsal, son los principales estabilizadores de esta articulación. Cada ligamento se halla constituido por una porción superficial y una porción profunda. Ambas porciones se originan en el cubito, la parte supeficial en la apófisis estiloides desde la base hasta la punta y la parte profunda en la fovea de la cabeza del cubito. Ambas porciones se insertan en la parte más distal de la fosa sigmoidea. Durante la supinación, la porción profunda del ligamento radiocubital palmar y la porción superficial del ligamento radiocubital dorsal se encuentran en tensión. Durante la pronación, la porción profunda del ligamento radiocubital dorsal y la porción superficial del ligamento radiocubital palmar son los que se hallan bajo tensión ${ }^{3-6}$.

La región central $(80 \%)$ del disco articular es avascular, en tanto que los ligamentos radiocubitales dorsal y palmar, como el $20 \%$ de la periferia del disco articular, presentan una rica vascularización por medio de ramas de la arteria interósea anterior, la arteria cubital, y las arterias medulares interóseas que penetran, a través de la cabeza cubital, en la región de la fóvea articular ${ }^{7}$. Como resultado de este patrón 
vascular, los desgarros periféricos del fibrocartílago triangular tienen un buen potencial de curación, mientras que la región avascular central tiene un potencial mínimo.

\section{DIAGNÓSTICO}

\section{Examen físico}

Algunas de las patologías de la ARCD son visibles a simple vista. El clásico ejemplo es el signo de la tecla de piano. Situando la palma de la mano en una superficie plana, se invita al paciente a presionar contra dicha superficie utilizando los músculos depresores del hombro, mientras el antebrazo se halla en pronación completa. Comparado con el lado contralateral, la elevación de la cabeza cubital en relación al radio, es considerado un signo positivo y sugiere una inestabilidad radio-cubital distal.

Tras identificar determinados parámetros anatómicos como la cabeza cubital y la apófisis estiloides, el piramidal, el pisiforme, la articulación semiluno-piramidal, el tendón del flexor capi ulnaris (FCU, cubital anterior), el tendón del extensor carpi ulnaris (cubital posterior, ECU) y su unión con el CFCT, se deben ubicar puntos de hipersensibilidad por medio de una palpación delicada de estas estructuras. La palpación siempre debe ser comparada con la extremidad contralateral.

Vale la pena mencionar dos maniobras específicas. En la primera, se le solicita al paciente colocar el codo sobre la mesa de examen, con el antebrazo perpendicular a la mesa. El examinador aplica un movimiento de cizallamiento entre el radio y el cúbito, con el antebrazo en pronación y supinación completas. La ausencia de desplazamiento entre ambos huesos en pronación y supinación completas, demuestra la integridad de los ligamentos radiocubitales palmar y dorsal, respectivamente. El comparar con el lado contralateral es crucial para determinar anormalidad. La aparición de dolor, durante estas maniobras, es sugestivo de una incongruencia en la ARCD o artritis de la misma.

Una segunda maniobra examina el efecto de la gravedad del lado cubital de la mano. Para esto se solicita al paciente mantenerse de pie con ambos codos flexionados a $90^{\circ}$. El examinador aplica una fuerza dirigida al suelo, en la mitad del antebrazo, mientras el paciente supina y prona alternativamente el mismo ${ }^{110}$. Esta maniobra aumenta la carga de la ARCD durante todo su rango de movimiento. Dolor en la ARCD durante esta maniobra indica patología de la misma.

\section{Radiografías estándar}

La evaluación radiográfica de la relación entre las superficies articulares del radio y el cúbito, o varianza cubital, es fundamental en el examen radiológico. La posición de la muñeca es importante para determinar el tipo de varianza cubital $^{9,10}$. La varianza puede cambiar 1 ó 2 $\mathrm{mm}$ durante el movimiento de prensión forzada, en pronación, comparada con la vista PA en neutro. Por lo tanto, $0^{\circ}$ de rotación en las vistas PA y prensión forzada en pronación, son dos vistas necesarias para evaluar la posición del cúbito en relación al radio (Figura 1). La varianza cubital positiva está asociada a la impactación cubito-carpiana, lesiones del ligamento

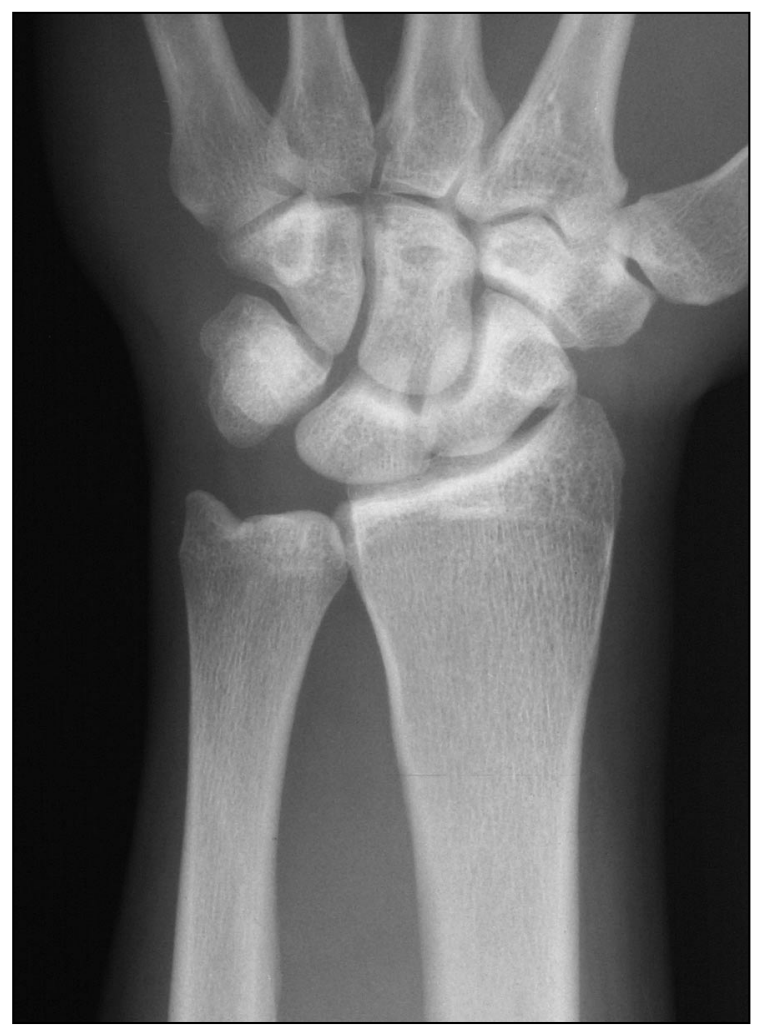

Figura 1. Configuración normal de la ARCD. 
semiluno-piramidal y desgarros del CFCT. La varianza negativa se halla implicada en la laxitud del los ligamentos carpianos y en la enfermedad de Kiënbock.

El segundo parámetro importante a evaluar en una radiografía PA es el ángulo de la ARCD y su congruencia. Raramente la escotadura sigmoidea y la superficie articular del cúbito son paralelas la una a la otra ${ }^{13}$. Normalmente se hallan inclinadas hacia la apófisis estiloides del cúbito, con un ángulo de aproximadamente $20^{\circ 1}$. Ocasionalmente, estas superficies articulares pueden ser paralelas al eje longitudinal del antebrazo (ARCD neutra), o pueden estar anguladas en sentido contrario (ARCD invertida). La configuración articular es especialmente importante en casos que requieren acortamiento cubital, puesto que el acortamiento cubital en el caso de ARCD invertida puede causar mayor choque entre la escotadura sigmoidea y la superficie articular del cúbito ${ }^{14}$. Signos radiológicos de artritis de la ARCD deberían ser específicamente investigados al realizar radiografías de rutina.

En una correcta vista lateral, el pisiforme debe encontrarse alineado con el tercio distal del escafoides. Radiografías oblicuas en posición de semipronación o semisupinación, pueden ser utilizadas para evaluar la articulación piso-piramidal y el gancho del ganchoso.

\section{Artrografía}

La artrografía de la muñeca con triple contraste es útil en el diagnóstico de desgarros del CFCT. Durante esta evaluación, se inyecta contraste en la articulación radiocarpal. 3 horas después, una segunda inyección se realiza en la ARCD y en la articulación mediocarpiana. La comunicación entre estos tres compartimientos es considerada positiva para un desgarro ligamentario. Diversos estudios realizados han demostrado resultados superiores con la triple inyección de contraste, en comparación con una artrografía con inyección simple ${ }^{15-17}$. Siempre deben correlacionarse los hallazgos radiológicos con la clínica del paciente. Muchos pacientes asintomáticos pueden presentar conexiones entre las filas proximal y distal del carpo. Herbert et al concluyeron que en el $74 \%$ de los pacientes evaluados bilateralmen- te con artrografía se encontró comunicación, entre las filas proximal y distal del carpo, en las muñecas contralaterales a la sintomática ${ }^{18}$. Cantor et al demostraron en 56 muñecas, que el $88 \%$ de los pacientes presentaba desgarros ligamentarios en las muñecas sintomáticas, tenía perforaciones bilaterales del CFCT $^{19}$.

El curso natural de los cambios degenerativos en el lado cubital de la muñeca y la existencia de comunicación entre compartimientos, por lo general dificulta la interpretación de los hallazgos. Es crítico entender los cambios del FCT relacionados con la edad, así como aquellos observados en la fila distal del carpo, y las articulaciones radio y cubito-carpiana ${ }^{20,21}$. La combinación de artrografía con medio de contraste aumenta la habilidad diagnóstica de las diferentes patologías.

\section{Tomografía computadorizada}

La reconstrucción tridimensional de imágenes adquiridas por medio de la tomografía computadorizada (TAC), facilitan el planeamiento preoperatorio, especialmente en casos de fracturas que involucran la ARCD. La TAC es también útil para la evaluación de la congruencia articular entre el radio y el cúbito, especialmente durante la osteoartritis temprana. Se han descrito diversos métodos para el diagnóstico de la subluxación radiocubital. De ellos el método del epicentro nos parece el más sensible. Es importante obtener imágenes en posición neutra, en supinación y pronación máximas ${ }^{23}$. Imágenes bajo estrés pueden evidenciar sutiles signos de subluxación ${ }^{24}$.

\section{Resonancia magnética nuclear (RMN)}

Fracturas ocultas, condromalacia, defectos osteocondrales y desgarros del CFCT son comúnmente diagnosticadas utilizando RMN. Estudios comparando la validez del uso de la RMN en el diagnóstico de los desgarros del FCT, en comparación con la artroscopia, revelaron un $90 \%$ de certeza con la $\mathrm{RMN}^{25}, 26$.

\section{Gammagrafía ósea}

La gammagrafía de tres fases resulta muy útil en pacientes con radiografías negativas ${ }^{27}$. 
Fracturas, infección, hematomas subcapsulares e inflamación, mostrarán captación aumentada en las tres fases. La gammagrafía tiene una alta sensiblidad pero baja especificidad, de manera que la correlación radiográfica es siempre necesaria.

\section{CLASIFICACIÓN Y MANEJO DE LOS PROBLEMAS DE LA ARCD}

Los problemas de la ARCD pueden ser clasificados en 3 grupos:

\section{a. Choque}

La impactación cubital, choque cubito-semilunar, carga cubito-carpiana, etc, son diversos términos utilizados para describir un mismo proceso crónico, degenerativo, causado por la carga excesiva a través del lado cubital de la mu$\tilde{n ̃ e c a}^{28}$. El término «choque cubito-carpiano», describe el mecanismo fisiopatológico, del choque entre el cúbito y el carpo.

La transferencia de la carga a través de la articulación de la muñeca con varianza neutra y en posición neutra, es acarreada por el radio $(82 \%)$ y por el cúbito $(18 \%)^{29}$. La magnitud de esta transferencia de la carga es afectada por la varianza cubital y por la inclinación palmar del radio distal. Un incremento de $2.5 \mathrm{~mm}$ de la varianza cubital, aumenta la carga cubito-carpiana a un $40 \%$. Cualquier factor que aumente la longitud del cúbito en relación al radio, da como resultado impactación de los huesos carpianos contra el cúbito y el disco articular. El impacto repetido de la cabeza cubital contra el fibro cartílago triangular (FCT) conlleva la perforación del mismo, la condromalacia de la cabeza cubital y desgarros del ligamento semiluno-piramidal ${ }^{28}$. Esta forma puede también ser dinámica y los síntomas solo presentarse durante la prensión forzada y la pronación del antebrazo.

Cambios relativos a la edad se observan en el 53\% de los pacientes mayores de 60 años, manifestándose como perforaciones en el $\mathrm{CFCT}^{20}$. Diversos estudios han identificado la asociación entre la perforación del FCT, la varianza cubital positiva y desgarros del ligamen- to semiluno-piramidal. Palmer y Werner mostraron que el $73 \%$ de los especimenes estudiados que presentaban perforaciones del FCT, presentaban asociada una varianza cubital positiva o neutra, y en la mayoría de los casos, coincidía con un desgarro del ligamento semiluno-piramidal $^{2}$. Su clasificación está universalmente aceptada (Tabla I) ${ }^{21}$.

Radiográficamente, se han identificado dos tipos de choque cúbito-carpiano: 1 . El choque cúbito-semilunar, entre la superficie articular cubital y el semilunar. 2. Choque cubito-piramidal, entre la apófisis estiloides cubital y el piramidal. El choque cubito-piramidal está comúnmente asociado a la varianza cubital neutra o positiva, pero puede también presentarse en la varianza negativa ${ }^{30}$. Comúnmente asociada a varianza cubital positiva congénita, consolidación viciosa de fracturas del radio distal, resección de la cabeza del radio o cierre prematuro de la epífisis distal del radio de cualquier origen. El choque cúbito-piramidal es independiente de la varianza cubital. Puede ocurrir debido a una consolidación viciosa de la apófisis estiloides cubital o secundario a una apófisis estiloides larga. El mecanismo involucra la impactación de la estiloides cubital contra el polo proximal del piramidal. La longitud relativa de la estiloides cubital se calcula usando el índice de la apófisis estiloides cubital ${ }^{14}$. Una estiloides excesivamente larga tiene una longitud total mayor a $6 \mathrm{~mm}^{31}$. La continua impactación puede generar condromalacia piramidal $y$, eventualmente, inestabilidad semiluno-piramidal ${ }^{32}$.

Independientemente de la localización del lugar de impactación, las manifestaciones clínicas incluyen dolor agudo o crónico en la región cubital de la muñeca, dolor exacerbado por la actividad, inflamación y limitación del movimiento de la muñeca y el antebrazo. Las opciones de tratamiento cambian considerablemente de acuerdo al tipo de choque cubital (Tabla II).

Como primer paso, se recomienda la modificación de la actividad, uso de férulas y medicación anti-inflamatoria. En caso de fracaso, se recomienda la intervención quirúrgica ${ }^{33}$.

La meta del tratamiento quirúrgico es la de disminuir la transferencia de la carga, a través del aspecto cubital de la muñeca. Si la etiología es una consolidación viciosa tras una frac- 
Tabla I - Lesiones del CFCt según Palmer

\begin{tabular}{|cl|}
\hline Clase & Descripción \\
\hline I & Lesión traumática \\
\hline IA & Perforaciones Centrales \\
\hline IB & Avulsión cubital sin o con fractura de la estiloides distal \\
\hline IC & Avulsión distal (ligamentos cubito-carpianos) \\
\hline ID & Avulsión radial sin o con fractura de la escotadura sigmoidea \\
\hline II & Lesión degenerativa \\
\hline IIA & Desgaste del FCT \\
\hline IIB & Desgaste del FCT, condromalacia cubital o semilunar \\
\hline IIC & Perforación del FCT, condromalacia cubital o semilunar \\
\hline IID & $\begin{array}{l}\text { Perforación del FCT, condromalacia cubital o semilunar, perforación del ligamento } \\
\text { semiluno-piramidal }\end{array}$ \\
\hline IIE & $\begin{array}{l}\text { Perforación del FCT, condromalacia cubital o semilunar, perforación del ligamento } \\
\text { semiluno-piramidal, artritis cubito-carpiana. }\end{array}$ \\
\hline
\end{tabular}

tura del radio distal, se deberá corregir para restablecer la congruencia articular de la ARCD. En ausencia de consolidación viciosa, una varianza cubital positiva puede ser tratada de dos diferentes formas (Tabla II).

El acortamiento cubital supone una osteotomía y resección de un segmento cubital en su tercio distal. La técnica, originalmente descripta por Milch ${ }^{34}$ en 1941, ha sido modificada desde entonces ${ }^{35-38}$. Las ventajas de esta técnica incluyen la descompresión efectiva del aspecto cubital de la muñeca, tensado de los ligamentos extrínsecos cubito-carpianos, promoviendo una mayor estabilidad semiluno-piramidal. Este procedimiento tiene un elevado porcentaje de consolidación y de éxito ${ }^{39}$. El tiempo promedio para la consolidación, en diferentes estudios realizados, varía de 7 a 21 semanas. Más del 95\% de los pacientes en distintas series publicadas, tiene resultados buenos o excelentes ${ }^{35-37,40-43}$. Las posibles complicaciones incluyen hasta un $4 \%$ porcentaje de pseudoartrosis, dolor con el movimiento, necesidad de extraer la placa de osteosíntesis y cambio en la configuración articular que conlleve a la incongruencia de la ARCD.
Un segundo tratamiento es la intervención del «wafer», en el que se resecan, de forma abierta o por artroscopia, los 2-4 $\mathrm{mm}$ de la cabeza cubital ${ }^{28,44,45}$. Esta técnica está recomendada en pacientes con impactación cubito-carpiana, Posibles complicaciones incluyen una inadecuada resección del cúbito y tendinitis del extensor cubital de la muñeca y su ruptura. El procedimiento está contraindicado en pacientes con inestabilidad o con cambios degenerativos de la ARCD, o en aquellos que requieren una resección mayor a $4 \mathrm{~mm}$ de acortamiento cubital. El número de series publicadas hasta la fecha, es limitado ${ }^{46-50}$ y a nosotros no nos gusta, porque al extirpar la cúpula del cúbito se crea una superficie con filo que se mueve contra la escotadura sigmoidea. No obstante en estudios comparativos ${ }^{50}$ Los autores concluyen que el procedimiento de wafer provee adecuado alivio del dolor sin la potencial complicación de una pseudoartrosis o necesidad de remover osteosíntesis.

\section{b. Inestabilidad}

La inestabilidad se define como un patrón anormal de contacto articular, durante o al fi- 


\section{Tabla II - Opciones DE TRATAMIENTO DE LA IMPACTACIÓN}

\begin{tabular}{|l|l|}
\hline \multicolumn{1}{|c|}{ I. Impactación cúbito-semilunar } & \multicolumn{1}{c|}{ Opciones de tratamiento } \\
\hline $\begin{array}{l}\text { a. Varianza cubital positiva secundaria a } \\
\text { consolidación viciosa del radio distal }\end{array}$ & $\begin{array}{l}\text { Osteotomía correctiva para corregir altura e } \\
\text { inclinación radial Y/O } \\
\text { - Osteotomía de acortamiento cubital }\end{array}$ \\
\hline $\begin{array}{l}\text { b. Varianza cubital positiva (neutra) sin } \\
\text { incongruencia de la ARCD }\end{array}$ & $\begin{array}{l}\text { - Osteotomía de acortamiento cubital } \\
\text { - Procedimiento de Wafer (artroscópico vs. } \\
\text { abierto) }\end{array}$ \\
\hline $\begin{array}{l}\text { c. Varianza cubital positiva (neutra) con } \\
\text { incongruencia de la ARCD }\end{array}$ & $\begin{array}{l}\text { - Procedimientos ablativos (ver tabla 4) } \\
\text { - Artroplastias de implante de la ARCD } \\
\text { (ver tabla 4) }\end{array}$ \\
\hline II. Impactación Estilo-piramidal & - Resección del fragmento de la estiloides \\
\hline a. Pseudoartrosis de la estiloides & - Fijado del FCT, artroscópico o abierto \\
\hline b. Apófisis estiloides larga & - Osteotomía de acortamiento \\
\hline
\end{tabular}

nal del rango de movimiento de la misma. Esto es debido a una alteración de la superficie articular, a una deficiencia de los ligamentos de sujeción, o a una combinación de ambas causas. La inestabilidad, subluxación y luxación de la ARCD son definidas en base a la relación espacial de la cabeza cubital con el radio y la unidad radio-carpiana. Si el cúbito es prominente dorsalmente, se denomina luxación cubital posterior de la ARCD, y si dicha prominencia ocurre en sentido palmar, se la denomina como luxación palmar cubital de la $\mathrm{ARCD}^{33}$.

Las luxaciones o subluxaciones de la ARCD pueden ser clasificadas en agudas o crónicas (Tabla III). Las luxaciones agudas pueden ocurrir como una lesión aislada ${ }^{51-54}$, o ser el resultado de fracturas de la cabeza del radio, fracturas del radio distal y fracturas de ambos huesos del antebrazo ${ }^{55-59}$. En las lesiones aisladas, las luxaciones cubitales dorsales ocurren como resultado de una hiperpronación, mientras que las luxaciones cubitales palmares ocurren por hipersupinación.

En las lesiones agudas, el aspecto clínico de las lesiones varía dependiendo de la presencia, o no, de lesiones asociadas ${ }^{60-61}$. En una fractura, la deformidad observada es por lo general aparente y requiere de un examen radiológico exhaustivo del antebrazo. Si la ARCD subluxada es una lesión aislada, solo es evidente la inflamación $^{33}$. Los movimientos de la muñeca suelen ser dolorosos, pero los movimientos del codo son normales, a menos de que coexista con una fractura de esta articulación. La rotación del antebrazo se halla siempre restringida. La pronación se encuentra limitada en las luxaciones palmares, mientras que la supinación se halla limitada en las luxaciones dorsales ${ }^{33}$. Las radiografías simples deben incluir dos vistas del antebrazo incluyendo el codo y la muñeca. Una radiografía lateral pura es esencial para detectar una subluxación o luxación en cualquier dirección ${ }^{62}$.

Los tipos de luxación de la ARCD pueden ser simples y complejas (Tabla III). Las luxaciones simples se reducen espontáneamente o con simples maniobras cerradas que requieren mínimo esfuerzo. Las luxaciones complejas, incluyen las luxaciones reducibles, las irreducibles y las inestables. La irreductibilidad de la 
luxación de la ARCD ha sido bien descrita. Por lo general es debida a interposición de los ligamentos del FCT, al extensor del meñique, al tendón del cubital posterior y a los extensores del anular y del meñique, tras su ruptura ${ }^{57,63-}$ 65. La mayoría de las luxaciones de la ARCD son producidas por impactos de alta energía, y se hallan frecuentemente asociadas a fracturas de la apófisis estiloides cubital ${ }^{66}$. El diagnóstico, en estos casos, depende del examen físico y de los hallazgos en la radiografía simple ${ }^{67-69}$. En casos de fracturas de la estiloides cubital, que afecten a más del $50 \%$ de la longitud de la apófisis estiloides y/o su desplazamiento mayor a $2 \mathrm{~mm}$, asociadas a fracturas del radio distal, el riesgo de inestabilidad de la ARCD se encuentra aumentado ${ }^{70}$.

El tratamiento de la subluxación/luxación simple aguda, consiste en la reducción e inmovilizacióndurante 6 semanas en un yeso braquioantebraquialpalmar. La posición del antebrazo debe ser neutra ya que los ligamentos se hallan laxos en esta posición.. Si se requiere de maniobras para la reducción cerrada, estas deben ser realizadas bajo anestesia regional, para una adecuada relajación muscular. Si tras la reducción la ARCD permanece inestable, mantenemos la reducción con agujas de Kirschner percutáneas. En las luxaciones subagudas no tratadas, los intentos de reducción están basados en la dirección de la luxación. Se han publica- do reducciones exitosas hasta 2 meses después en las luxaciones dorsales, pero tan solo de 3 semanas en las luxaciones palmares ${ }^{71}$. La reducción abierta está indicada cuando la reducción cerrada no es posible, o insatisfactoria. Sin embargo, si la estiloides cubital se encuentra fracturada, esta debería ser estabilizada por medio de una aguja de Kirschner, una banda de tensión, o un cerclaje intraóseo ${ }^{72}$. Si la estiloides cubital se encuentra intacta, pero existe un desgarro del CFCT, este debe ser reinsertado al cúbito. La posición y el tiempo de la inmovilización postoperatoria son similares al de las luxaciones simples.

La inestabilidad crónica de la ARCD puede ocurrir aislada o hallarse asociada a consolidación viciosa de fracturas del radio distal. En ausencia de consolidación viciosa, la inestabilidad crónica puede ser tratada por medio de la reconstrucción de tejidos blandos (Tabla 3). Sin embargo, los siguientes criterios deben cumplirse antes de intentar cualquier reconstrucción de tejidos blandos: 1. ARCD congruente, 2. ligamentos radiocarpianos estables, 3. ligamentos cubito-carpianos estables. Si no existe una estabilidad satisfactoria entre el radio y el carpo, o entre el cúbito y el carpo, se debe intentar estabilizar estas estructuras, como primera medida. Si la ARCD no es congruente, los procedimientos para la reconstrucción de tejidos blandos no serán satisfactorios.

\section{Tabla III - Opciones DE TRATAMIENTO PARA LA INESTABILIDAD DE LA ARCD}

\begin{tabular}{|c|c|c|}
\hline \multicolumn{2}{|c|}{ Tipo de Inestabilidad } & Opciones de Tratamiento \\
\hline \multirow[b]{2}{*}{ Aguda } & Simple $\left(^{*}\right)$ & $\begin{array}{l}\text { - Reducción cerrada } \\
\text { - Inmovilización con yeso }\end{array}$ \\
\hline & Compleja $\left(^{*}\right)$ & $\begin{array}{l}\text { - Reducción Cerrada vs Abierta con fijación } \\
\text { con agujas de Kirschner } \\
\text { - Inmovilización con yeso }\end{array}$ \\
\hline \multicolumn{2}{|l|}{ Crónica } & $\begin{array}{l}\text { - Reconstrucción de tejidos blandos } \\
\text { - Intra-articular: Scheker }{ }^{78}, \text { Adams }^{79} \\
\text { - Extra-articular } \\
74-77\end{array}$ \\
\hline
\end{tabular}

${ }^{\star}{ }^{*}$ Ver texto para la descripción de la Inestabilidad simple y compleja. 


\section{Tabla IV - Opciones DE TRATAMIENTO PARA LA INCONGRUENCIA/ ARTHRITIS DE LA} ARCD

\begin{tabular}{|l|l|}
\hline Tipo de Artritis & Opciones de Tratamiento \\
\hline Osteoartritis temprana & - Osteotomía de acortamiento cúbito ${ }^{84}$ \\
\hline Osteoartritis establecida & - Procedimientos ablativos: Sauve-Kapandji90, HIA ${ }^{* 98}$, \\
& resección distal congruente de la ulna distal ${ }^{99}$, Darrach $^{81}$ \\
& - Artroplastias de implante: \\
& - Hemi-arthroplastia (Swanson ${ }^{105}$, Herbert and van \\
& Schoonhoven ${ }^{107}$, Berger $\left.^{108}\right)$ \\
& - Arthroplastia Total $\left(\right.$ Scheker $\left.^{109}\right)$ \\
\hline
\end{tabular}

(*) HIA: Arthroplastia de Hemi-resección e Interposition.

Inicialmente, la inestabilidad de la ARCD es dinámica, progresando luego, a la inestabilidad estática con el avance de la osteoartritis. Esta progresión, de dinámica a estática, se realiza en cuatro etapas $^{73}$. En la etapa 1, el paciente tiene la sensación subjetiva de que su muñeca cede. No hay signos clínicos objetivos, ni radiológicos. Denominada inestabilidad dinámica. Etapa 2, los síntomas son similares a los de la etapa 1, pero la articulación puede ser luxada o subluxada objetivamente. Etapa 3, la limitación del movimiento y el dolor son más severos. La articulación descansa en una posición inestable, pero puede ser reducida. La subluxación y el mal alineamiento son aparentes en las radiografías simples. A partir de este punto, la inestabilidad se ha vuelto estática. Etapa 4, la limitación del movimiento es la característica predominante y la deformidad fija se halla establecida. Todo esto conlleva un alto riesgo de desarrollo de osteoartritis. La estabilidad articular se mantiene en base a la función de la integridad de los ligamentos y a la alineación o congruencia de la articulación radiocubital. Bowers ha clasificado la inestabilidad de la ARCD en 4 gru$\operatorname{pos}^{73}$. Grupo 1, defecto primario en los ligamentos. Grupo 2, deficiencias intra-articulares de la congruencia articular con pérdida de la tensión de los ligamentos. Grupo 3, combinación de patología tanto de los ligamentos como de la superficie articular. Grupo 4, la deficiencia ligamentaria se encuentra asociada a problemas extraarticulares, como a una consolidación viciosa de la metáfisis distal del radio.

\section{CONGRUENCIA DE LA ARCD CON INESTABILIDAD CRÓNICA}

Se han descrito dos técnicas para proporcionar estabilidad a la ARCD: I. Técnica Extraarticular, y II. Técnica Intraarticular. Las reconstrucciones extraarticulares. Estas técnicas no restablecen la anatomía ligamentaria de la ARCD. La reconstrucción extraarticular puede ser obtenida por medio de una técnica directa $\mathrm{de}^{74}$, o a una técnica indirecta de estabilización radiocubital a través de una tenodesis cubitocarpiana $^{75-77}$. Estas técnicas no proporcionan suficiente estabilidad a la ARCD.

Las reconstrucciones intra-articulares, más complejas técnicamente, han sido diseñadas para reconstruir la anatomía ligamentaria del $\mathrm{FCT}^{73,78-80}$. En su artículo inicial, Scheker mostraba desaparición deldolordel dolor en 12 de 14 pacientes operados. Solo dos de ellos referían molestias durante el levantamiento de pe$\operatorname{sos}^{78}$. En todos la pronación y supinación fueron satisfactorias $\left(80^{\circ} / 82^{\circ}\right)$ tras la intervención. Adams et al, hicieron un seguimiento de 14 pacientes durante 2,2 años. Nueve de los 14 pacientes tuvieron un alivio total del dolor, con una pronación/supinación media de de $72^{\circ}$ y $70^{\circ}$, respectivamente ${ }^{79}$.

\section{INCONGRUENCIA/ARTRITIS}

La incongruencia de la ARCD se caracteriza por artritis de la superficie articular del cú- 
bito o la escotadura sigmoidea del radio. Frecuentemente ocurre tras fracturas del radio distal, osteoartritis, inestabilidad crónica, infección o artritis reumatoidea. La alteración de la ARCD producida por la incongruencia de las superficies articulares ha sido tradicionalmente tratada por medio de la resección parcial o total de la cabeza cubital (Tabla IV) ${ }^{81-83}$. Estos procedimientos ablativos no restauran la biomecánica del antebrazo, pero proveen alivio del dolor y grados variables de función útil, con sus consecuencias adversas. En casos selectos de osteoartritis temprana, donde solo el tercio proximal de la ARCD se encuentra comprometido, un acortamiento cubital puede ser beneficioso, ya que cambia el punto de contacto entre la escotadura sigmoidea del radio y la cabeza cubital (Figura 2). Utilizando esta técnica, Scheker mostraba un alivio del dolor en 16 de 32 pacientes con un seguimiento promedio de 38 me$\operatorname{ses}^{84}$. La persistencia de dolor severo que requirió algún procedimiento ablativo fue necesario en 7 casos.

Para el manejo de la artritis instaurada en la ARCD, el más antiguo y conocido de estos pro-

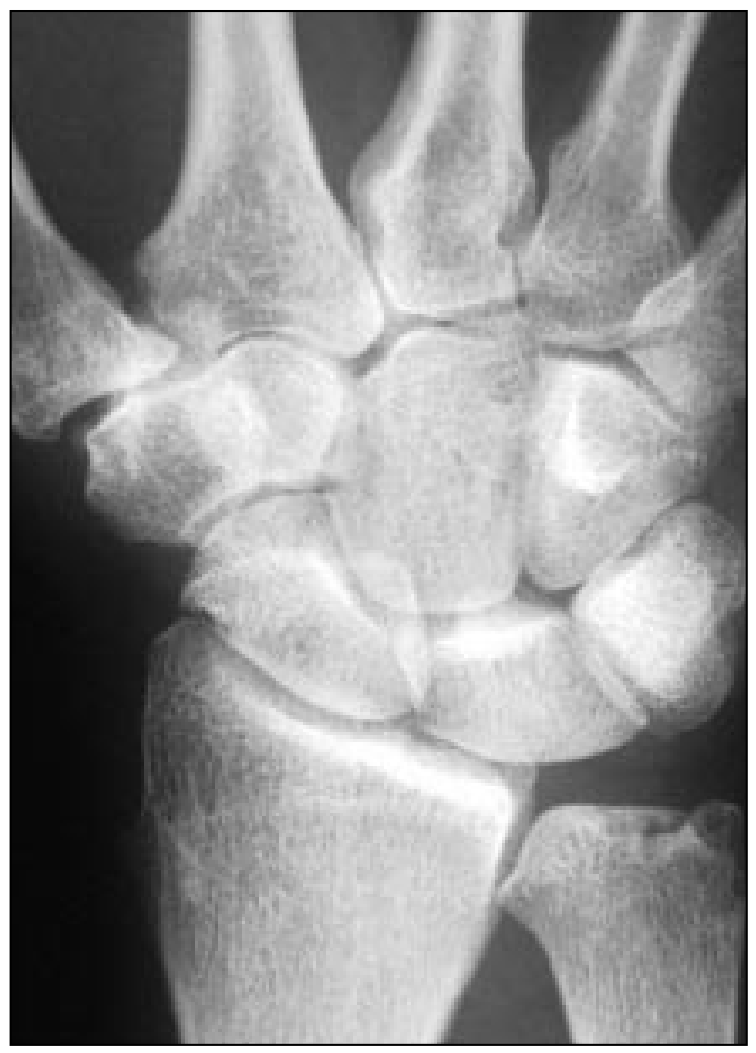

Figura 2. Osteoartritis temprana. cedimientos es la resección total de la cabeza cubital, o procedimiento de Darrach ${ }^{81}$. Resecando la cabeza cubital, el procedimiento de Darrach elimina efectivamente el dolor asociado con la artritis de la ARCD. Aunque las publicaciones iniciales con esta técnica mostraron éxito en más de $80 \%$ de los pacientes estudia$\operatorname{dos}^{85,86}$, artículos recientes en grupos mayores de pacientes, han demostrado un porcentaje alto de fracasos, resultando en una seria discapacidad, especialmente en pacientes jóvenes ${ }^{87,88}$.

El procedimiento, en sí mismo, conlleva la inestabilidad radiocubital, ya que se resecan la cabeza del cúbito, el CFCT, los ligamentos radiocubitales y la vaina del tendón del cubital posterior, dejando un antebrazo completamente inestable ${ }^{87,88}$. Lees y Scheker demostraron esta inestabilidad convergente del cúbito tras Darrach al levantar tan sólo 2,26 kilos ${ }^{111}$. La presencia de inestabilidad radiocubital se manifiesta con dolor y chasquido del muñón del cúbito (Figura 3). Con la pérdida del soporte cubital de la muñeca, la translocación cubital del carpo es inevitable ${ }^{89}$. Un extremo romo del cúbito, puede también ser causa de ruptura de los tendones extensores ${ }^{85}$.

Otro de los procedimientos comúnmente utilizado es el Sáuve-Kapandji, que consiste en la fusión de la cabeza cubital al radio distal y la creación de una pseudo artrosis del cúbito ${ }^{90}$. El preservar la cabeza cubital tiene la ventaja de mantener el soporte cubital del carpo, el CFCT y el tendón del cubital posterior. Esta técnica se halla indicada en pacientes jóvenes, con intensa utilización de sus muñecas, y en pacientes con incompetencia ligamentaria radiocarpiana como en la artritis reumatoidea ${ }^{95}$ Aunque Sanders ${ }^{91}$, Mikkelsen ${ }^{92}$, Vincent ${ }^{93}$ y Rothwell ${ }^{94}$ mostraron resultados satisfactorios, en otros estudios se ha visto una alta incidencia de inestabilidad del muñón cubital remanente con dolor, y así como la osificación heterotópica en la pseudoartrosis con pérdida de la prono-supinación ${ }^{92,}$ 93, 96, 97.

Otros procedimientos más conservadores como la artroplastía de hemiresección e interposición (AHI), introducida por Bowers ${ }^{98}$, y la resección cubital distal congruente, descripta por Watson ${ }^{99}$ tienen similares inconvenientes, es decir, la inestabilidad radial resultante y su cho- 


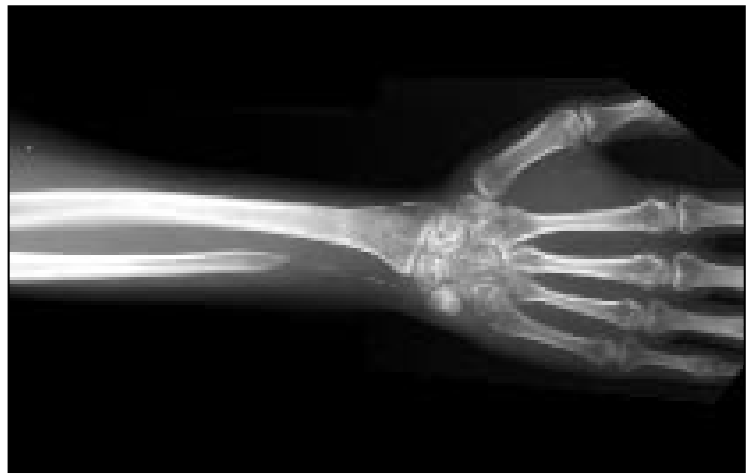

Figura 3a. Reseccion cubital con reconstrucción ligamentaria.

que contra el cúbito en la carga. Aunque los resultados de estos procedimientos pueden ser satisfactorios en pacientes con muñecas a las que se les demanda poca actividad, los resultados con frecuencia no son buenos en individuos activos $^{98-103 .}$.

Una alternativa final en la artritis de la ARCD es el reemplazo protésico de la misma. Esta puede ser separada en dos grupos: la hemiartroplastia y la artroplastiaartroplastía total de la ARCD.

\section{HEMIARTROPLASTIA DE LA ARCD}

En el mercado hay tres diferentes diseños de prótesis para la hemiartroplastia. El implante de Swanson, que consiste en una cabeza cubital protésica de silicona con un vástago intramedular redondo de metal ${ }^{105}$. Los resultados a largo plazo demuestran un alivio efectivo del dolor, con un $40 \%$ de inclinación de la prótesis debido a reabsorción y $15 \%$ de fracturas del implante $^{106}$. La cabeza de cerámica recomendada por Herbert y van Schoonhoven ${ }^{107}$, no tiene medio de fijación alguno al CFCT, por lo tanto, la estabilidad de la ARCD depende de la estabilidad del CFCT. En un seguimiento a 27 meses, los autores reportan reducción del dolor, mejoría de la fuerza de prensión y del rango de movimiento, aunque en $2 / 23$ requirieron reintervención por inestabilidad. Todos los pacientes presentaron reabsorción ósea de 1 ó $2 \mathrm{~mm}$ por debajo del cuello de la prótesis con el subsiguiente aflojamiento de la misma en un pacien-

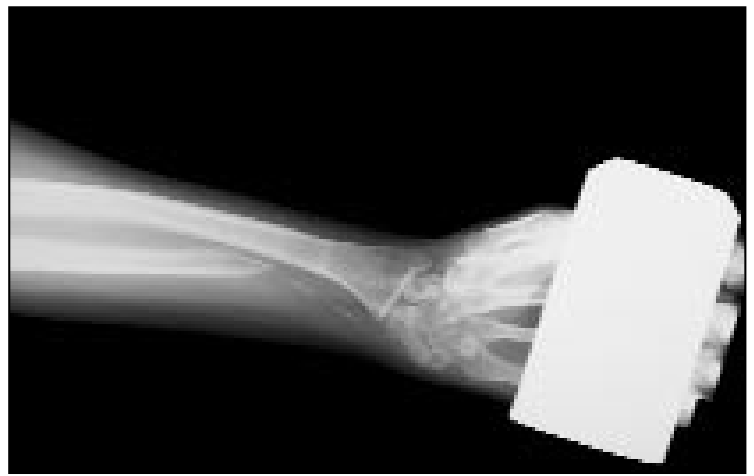

Figura $3 b$. Levantado $2,26 \mathrm{~kg}$ de peso en posición neutra ponen en evidencia el choque radiocubital.

te. El último ejemplo de hemiartroplastia fue desarrollada por Berger y consiste en una cabeza esférica compuesta de una aleación de cromo-cobalto ${ }^{108}$. No han sido reportadas complicaciones. Radiográficamente, una reabsorción universal leve se observa en el cubital, inmediatamente proximal al cuello del dispositivo. Este modelo permite la estabilización del CFCT y de la vaina del cubital posterior por medio de suturas. Requiere la presencia una escotadura sigmoidea congruente y puede no ser adecuada para aquellos casos en los cuales se ha realizado una amplia resección cubital.

\section{ARTROPLASTÍA TOTAL DE LA ARCD}

Finalmente, la única opción para una artroplastía total de la ARCD ha sido descrita por Scheker ${ }^{109}$. Este modelo de implante consiste en una prótesis semi-constreñida y modular. Consta de un componente cubital, un vástago endomedular construido en una aleación de cromo-cobalto recubierto con plasma de titanio, una placa radial, y una bolilla de polietileno de ultra alto peso molecular (Figura 4). Los componentes reemplazan el cúbito distal (vástago y cabeza cubital), la escotadura sigmoidea del radio (placa radial) y el fibrocartílago triangular (cubierta de la placa radial). El rociado con plasma de titanio del vástago cubital produce una superficie altamente porosa la cual permite la osteointegración del implante al cúbito, promoviendo una mejor fijación del implante. Esta prótesis ha sido diseñada para mantener una pre- 


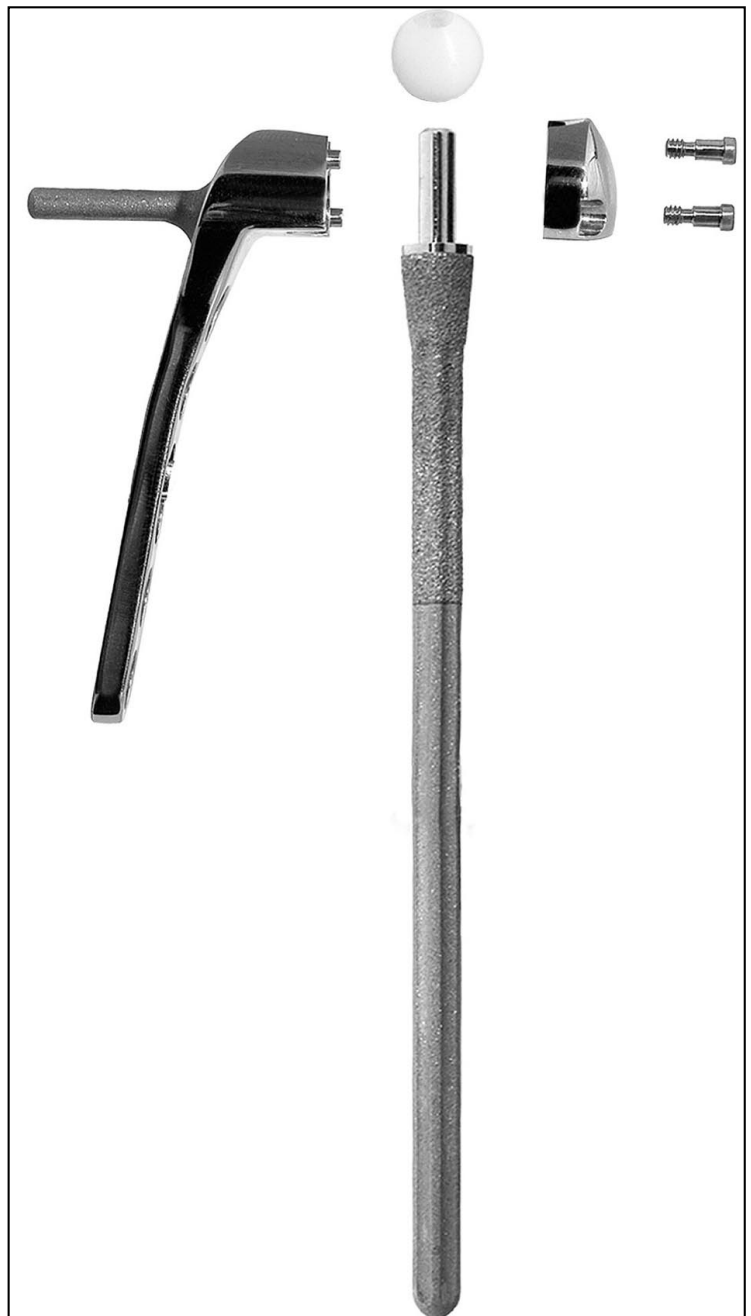

Figura 4. Prótesis Scheker para reemplazo total de la ARCD.

cisa relación anatómica entre el radio distal y el cúbito. (Figura 5). Permite una migración longitudinal del radio durante la pronación y supinación del antebrazo, a la vez que reestablece la capacidad de levantar peso al reconstruir el soporte cubital al radio distal. A la fecha, 140 pacientes han sido sometidos a reemplazo total de la ARCD con este implante. Resultados a medio y largo plazo (seguimiento promedio de 5,9 años) con esta prótesis han demostrado que todos los pacientes han mejorado la fuerza de prensión y levantamiento de peso (promedio 6,4 kilos) sin dolor. La evaluación por medio del DASH ${ }^{112}$ y el PRWE ${ }^{113}$ por parte de los pacientes fue de 23.1 y 30.1 , respectivamente. Todos los pacientes experimentaron la desaparición o una notoria disminución del dolor durante el movimiento. Por medio de

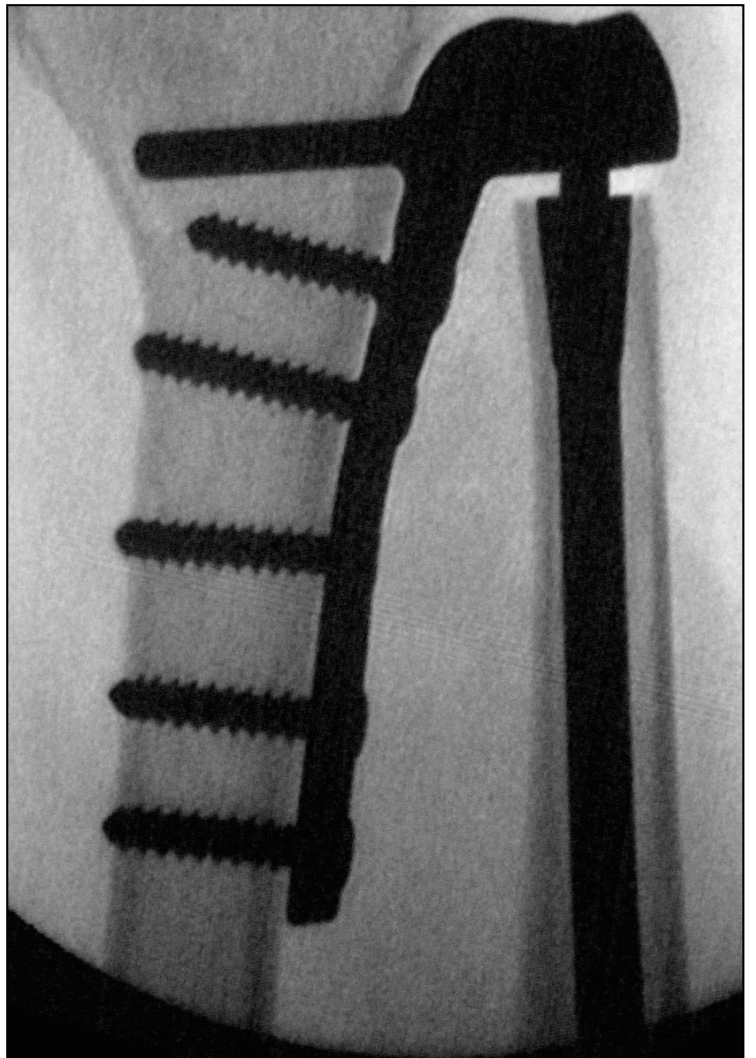

Figura 5. Imagen intraoperatoria del implante.

evaluaciones funcionales subjetivas por parte del paciente, se determinó que el resultado era superior ${ }^{114,115,116}$, al resultado con procedimientos ablativos del cúbito distal. La fuerza de prensión, en el lado intervenido, mejoró a un promedio del $67 \%$ de la fuerza del lado contralateral. Significativamente, todos los pacientes recobraron la capacidad de levantar peso, con la mano del lado intervenido, con la muñeca en posición neutra, al reestablecerse el soporte del radio distal (Figura 6). Esto permitió eliminar, o disminuir en forma importante, el dolor secundario al pellizcamiento radiocubital durante estos esfuerzos. El $78 \%$ de los pacientes retornaron a sus tareas habituales.

\section{RADIOLOGÍA}

El seguimiento radiológico de los casos ha demostrado la ausencia de radiotransparencia alrededor del vástago cubital en todos los casos implantados, con la excepción de un caso en el cual el paciente sufrió una infección por Staphylococcus epidermidis. La radio transparencia es 


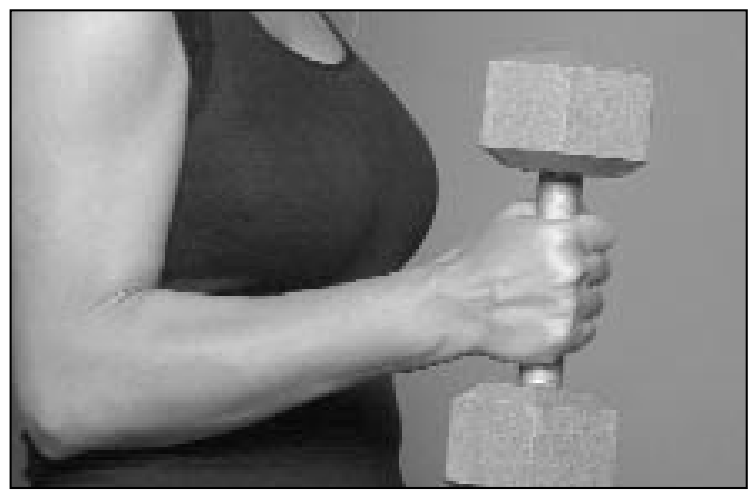

Fig. 6. La capacidad para levantar peso es reestablecida.

considerado un signo radiológico indirecto de aflojamiento del implante ${ }^{117}$.

\section{COMPLICACIONES}

Entre las pocas complicaciones observadas con este implante, cabe destacar 1 infección a
Staphylococcus epidermidis sufrida por 1 paciente. Se extrajo el implante, y tras antibioterapia sistémica y local, se sustituyó por otro implante sin más complicaciones. Un paciente desarrolló osificación heterotópica alrededor del vástago cubital, disminuyendo el rango de movimiento. Se resecó sin que haya habido recidiva. Finalmente, dos pacientes sufrieron fractura del vástago cubital en accidentes de tráfico, y requirieron su reemplazo. Ambos continúan con buena evolución.

En resumen, en este trabajo hemos hecho una revisión de los problemas que afectan a la radio-cubital distal. Es de esperar, que según mejore nuestro conocimiento de la anatomía y la biomecánica, y mejoren los materiales un número cada vez mayor de pacientes se beneficien del reemplazo protésico de la ARCD, pero lo que es más importante que en muchos pacientes se aborte la progresión a la artrosis por un mejor tratamiento de la patología original.

\section{BIBLIOGRAFÍA}

1. Af Ekenstam F, Haegert CG. Anatomical studies on the geometry and stability of the distal radioulnar joint. Scand J Plast Reconstr Surg 1985; 19:17.

2. Palmer AK, Werner FW. The triangular fibrocartilage complex of the wrist: anatomy and function. J Hand Surg 1981; 6A: 153-62.

3. Af Ekenstam FW, Palmer AK, Glisson RR. The load on the radius and ulna in different positions of the wrist and forearm: A cadaver study. Acta Orthop Scand 1984; 55: 363.

4. Schuind F, An KN, Berglund L, et al. The distal radioulnar ligaments: A biomechanical study. J Hand Surg 1991; 16A: 1106.

5. Haegert CG. Distal radius fracture and the distal radioulnar joint-anatomical considerations. Handchir Mikrochir Plast Chir 1994; 26:22.

6. Acosta R, Hnat W, Scheker LR. Distal radio-ulnar ligament motion during supination and pronation. J Hand Surg $[\mathrm{Br}]$. 1993;18(4):502-5.
7. Bednar MS, Arnoczky SP, Weiland AJ. The microvasculature of the triangular fibrocartilage complex: Its clinical significance. J Hand Surg 1991; 16A: 1101.

8. Hulten O. Uber anatomische varionen der hand-Gelenkknochen. Acta Radiol 1928; 9: 155.

9. Epner RA, Bowers WH, Guilford WB. Ulnar variance: the effect of wrist positioning and roentgen filming technique. $J$ Hand Surg (Am) 1982; 7: 298-305.

10. Palmer AK, Glisson RR, Werner FW. Ulnar variance determination. J Hand Surg 1982; 7: 376-79.

11. Friedman SL, Palmer AK, Short WH, Levinsohn EM, Halperin LS. The change in ulnar variance with grip. J Hand Surg (Am) 1993; 18: 713-716.

12. Tomaino MM. The importance of pronated grip x-ray view in evaluating ulnar variance. J Hand Surg (Am) 2000; 25: 352-357.

13. Sagerman SD, Zogby RG, Palmer AK, Werner FW, Fortino MD. Relative articular inclina- tion of the distal radioulnar joint: A radiographic study. J Hand Surg (Am) 1995; 20A: 597-601.

14. Tolat AR, Sanderson PL, DeSmet L, Stanley JK. The gymnast's wrist: acquired positive ulnar variance following epiphyseal injury. J Hand Surg 1992; 17B: 678-81.

15. Zinberg EM, Palmer AK, Coren $A B$ et al. The triple-injection wrist arthrogram. J Hand Surg (Am) 1988; 13: 803-809.

16. Belsole RJ, Quinn SF, Greene $T L$, et al. Digital subtraction arthrography of the wrist. J Bone Joint Surg Am 1990; 72: 846-851.

17. Levinsohn EM, Rosen ID, Palmer AK. Wrist arthrography: Value of three-compartment injection method. Radiology 1991; 179: 231-9.

18. Herbert TJ, Faithfull RG, McCann DJ, et al. Bilateral arthrography of the wrist. J Hand Surg (Br) 1990; 15: 233-5.

19. Cantor RM, Stern PJ, Wyrick JD, et al. The relevance of li- 
gament tears or perforations in the diagnosis of wrist pain: An arthrographic study. J Hand Surg (Am) 19:945-953, 1994.

20. Mikic ZD. Age changes in the triangular fibrocartilage of the wrist joint. J Anat 1978; 126: 367.

21. Palmer AK. Triangular fibrocartilage complex lesions: A classification. J Hand Surg 1989; 14A: 594.

22. Blair WF, Berger RA, El-Khoury GY. Arthrotomography of the wrist: An experimental and preliminary clinical study. J Hand Surg 1985; 10A: 350.

23. Wechsler RJ, Wehbe MA, Rifkin MD, et al. Computed tomography diagnosis of distal radioulnar subluxation. Skeletal Radiol 1987; 16: 1-5.

24. Pirela-Cruz MA, Goll SR, Klug $M$ et al. Stress computed tomography analysis of the distal radioulnar joint: a diagnostic tool for determining translational motion. J Hand Surg (Am) 1991; 16: 75-82.

25. Golimbu CN, Firooznia $\mathrm{H}, \mathrm{Me}-$ lone CP, et al. Tears of the triangular fibrocartilage of the wrist: MR imaging. Radiology 1989; 173: 731.

26. Zlatkin MB, Chao PC, Osterman $A L$ et al. Chronic wrist pain: Evaluation with high resolution MR imaging. Radiology 1989; 173: 723.

27. Shewing DJ, Savage R, Thomas G. Experience of the early use of technetium-99 bone scintigraphy in wrist injury. $\mathrm{J}$ Hand Surg 1994; 19: 114-7.

28. Palmer AK. Triangular fibrocartilage disorders: Injury patterns and treatment. Arthroscopy 1990; 6: 125-132.

29. Palmer AK, Werner FW. Biomechanics of the distal radioulnar joint. Clin Orthop 1984; 187: 26-35.

30. Tomaino MM. Ulnar impaction syndrome in the ulnar negative and neutral wrist: diagnosis and pathoanatomy. J Hand Surg (Br) 1998; 23: 754-757.

31. Garcia-Elias M. Dorsal fractures of the triquetrum: avulsion or compression fractures? J Hand Surg (Am) 1987; 12: 266-268.

32. Topper SM, Wood MB, Ruby LK. Ulnar styloid impaction syndrome. J Hand Surg (Am) 1997; 22: 699-704.

33. Bruckner JD, Alexander $\mathrm{AH}$, Lichtman DM. Acute dislocations of the distal radioulnar joint. Instr Course Lect 27-36.

34. Milch F.Cuff resection of the ulna for malunited Colles' fracture. J Bone Joint Surg 1941; 23: 311-13.

35. Wehbe MA, Cautilli DA. Ulnar shortening using the $A O$ small distractor. J Hand Surg (Am) 1995; 78: 348-356.

36. Rayhack JM, Gasser SI, Latta LL et al. Precision oblique osteotomy for shortening of the ulna. J Hand Surg 1993; 18: 908-918.

37. Darrow JC Jr, Linscheid RL, Dobyns JH et al. Distal ulnar recession for disorders of the distal radioulnar joint. $\mathrm{J}$ Hand Surg (Am) 1985; 10: 482-491.

38. Mizuseki T, Tsuge K, Ikuta Y. Precise ulna-shortening osteotomy with a new device. J Hand Surg (Am) 2001; 26: 931-9.

39. Friedman SL, Palmer AK. The ulnar impaction syndrome. Hand Clin 1991; 7: 295-310.

40. Boulas HJ. Milek MA. Ulnar shortening for tears of the triangular fibrocartilage complex. J Hand Surg (Am) 1990; 15: 415-420.

41. Chun S, Palmer AK. The ulnar impaction syndrome: Followup of ulnar shortening osteotomy. J Hand Surg 1993; 18: 46-53.

42. Chen NC, Wolfe SW. Ulna shortening osteotomy using a compression device. J Hand Surg [Am]. 2003; 28:88-93.

43. Minami $A$, Kato $H$. Ulnar shortening for triangular fibrocartilage complex tears associated with ulnar positive variance. J Hand Surg [Am]. 1998; 23: 904-8.

44. Feldon P, Terrono AL, Belsky MR. Wafer distal ulna resection for triangular fibrocartilage tears and/or ulna impaction syndrome. J Hand Surg (Am) 1992; 17: 731-737.

45. Wnorowski DC, Palmer AK, Werner FW, et al. Anatomic and biomechanical analysis of the arthroscopic wafer procedure. Arthroscopy 1992; 8: 204-212.

46. Nagle DJ, Bernstein MA. Laserassisted arthroscopic ulnar shortening. Arthroscopy. 2002 Nov-Dec;18(9):1046-51.

47. Tomaino MM, Weiser RW. Combined arthroscopic TFCC debridement and wafer resection of the distal ulna in wrists with triangular fibrocartilage complex tears and positive ulnar variance. J Hand Surg [Am]. 2001 Nov;26(6):1047-52.

48. Tomaino MM. Results of the wafer procedure for ulnar impaction syndrome in the ulnar negative and neutral wrist. J Hand Surg [Br]. 1999 Dec; 24(6): 671-5.

49. Constantine KJ, Tomaino MM, Herndon JH, Sotereanos DG. Comparison of ulnar shortening osteotomy and the wafer resection procedure as treatment for ulnar impaction syndrome. J Hand Surg [Am]. 2000 Jan; 25(1): 55-60.

50. Schuurman AH, Bos KE. The ulno-carpal abutment syndrome. Follow-up of the wafer procedure. J Hand Surg $[\mathrm{Br}]$. 1995 Apr;20(2):171-7.

51. Dell PC. Traumatic disorders of the distal radioulnar joint. Clin Sports Med 1992; 11:141-159.

52. Rainey RK, Pfautsch ML. Traumatic volar dislocation of the distal radioulnar joint. Orthopedics 1985; 8: 896-900.

53. Dameron TB Jr. Traumatic dislocation of the distal radioulnar joint. Clin Orthop 1972; 83: 55-63.

54. Milch H. So-called dislocation of the lower end of the ulna. Ann Surg 1942; 116:282-292.

55. Edwards GS Jr, Jupiter JB. Radial head fractures with acute distal radioulnar dislocation: Essex-Lopresti revisited. Clin Orthop 1988; 234: 61-69.

56. Eglseder WA, Hay M. Combined Essex-Lopresti and radial 
shaft fractures:Case report. J Trauma 1993; 34: 310-312.

57. Bruckner JD, Lichtman DM, Alexander $\mathrm{AH}$. Complex dislocations of the distal radioulnar joint: Recognition and management. Clin Orthop 1992; 275: 90-103.

58. Porter ML, Tillman RM. Pilon fractures of the wrist: Displaced intra-articular fractures of the distal radius. $\mathrm{J}$ Hand Surg 1992; 17B: 63-68.

59. Goldberg HD, Young JW, Reiner $\mathrm{Bl}$ et al. Double injuries of the forearm: A common occurrence. Radiology 1992; 185: 223-227.

60. Braun RM. The distal joint of the radius and ulna: Diagnostic studies and treatment rationale. Clin Orthop 1992; 275: 74-78.

61. Drewniany JJ, Palmer AK. Injuries to the distal radioulnar joint. Orthop Clin North Am 1986; 17: 451-459.

62. Mino DE, Palmer AK, Levinhson EM. The role of radiography and computerized tomography in the diagnosis of subluxation and dislocation of the distal radioulnar joint. $J$ Hand Surg 1983; 8A: 23-31.

63. Itoh $\mathrm{Y}$, Horiuchi $\mathrm{Y}$, Takahashi M, et al. Extensor tendon involvement in Smith's and Galeazzi fractures. J Hand Surg 1987; 12A: 535-540.

64. Jenkins NH, Mintowt-Czyz WJ, Fairclough JA. Irreducible dislocation of the distal radioulnar joint. Injury 1987; 18: 40-3.

65. Hanel DP. Scheid DK. Irreducible fracture-dislocation of the distal radioulnar joint secondary to entrapment of the extensor carpi ulnaris tendon. Clin Orthop 1988; 234: 56-60.

66. Hauck RM, Skahen J III, Palmer AK. Classification and treatment of ulnar styloid nonunion. J Hand Surg 1996; 21A: 418-422.

67. Oskarsson GV, Aaser P, Hjall A. Do we underestimate the predictive value of the ulnar styloid affection in Colles fractures? Arch Orthop Trauma Surg 1997; 116: 341-344.
68. Stoffelen D, de Smet L, Broos P. The importance of the distal radioulnar joint in distal radial fractures. $J$ Hand Surg 1998; 23B: 507-511.

69. Geissler WB, Fernandez DL, Lamey DM. Distal radioulnar joint injuries associated with fractures of the distal radius. Clin Orthop 1996; 327: 135-146.

70. May M, Lawton JN, Blazar PE. Ulnar styloid fractures associated with distal radius fractures: Incidence and implications for distal radioulnar joint instability. J Hand Surg 2002; 27A: 965971.

71. Dameron TB, Jr. Traumatic dislocation of the distal radioulnar joint. Clin Orthop 1972; 83: 55-63.

72. Shaw JA, Bruno A, Paul EM. Ulnar styloid fixation in the treatment of posttraumatic instability of the radioulnar joint: $A$ biomechanical study with clinical correlation. J Hand Surg 1990; 15A: 712-720.

73. Bowers WH. The distal radioulnar joint. In: Green DP, Hotchkiss RN, Peterson WC, eds. Green's Operative Hand Surgery 4th ed. Philadelphia:Churchill Livingstone, 1999: 9861014.

74. Fulkerson JP, Watson HK. Congenital anterior subluxation of the distal ulna. A case report. Clin Orthop 1978; 131: 179-182.

75. Breen TF, Jupiter JB. Extensor carpi ulnaris and flexor carpi ulnaris tenodesis of the unstable distal ulna. J Hand Surg 1989; 14A: 612-617.

76. Hui FC, Linscheid RL. Ulnotriquetral augmentation tenodesis: a reconstructive procedure for dorsal subluxation of the distal radioulnar joint. $\mathrm{J}$ Hand Surg 1982; 7: 230-236.

77. Tsai T-M, Stilwell JH. Repair of chronic subluxation of the distal radioulnar joint (ulnar dorsal) using flexor carpi ulnaris tendon. J Hand Surg 1984; 9B: 289-293.

78. Scheker LR, Belliappa PP, Acosta R, German DS. Reconstruction of the dorsal liga- ment of the triangular fibrocartilage complex. J Hand Surg 1994; 19B: 310-8.

79. Adams BD, Berger RA. An anatomical reconstruction of the distal radioulnar ligaments for posttraumatic distal radioulnar joint instability. J Hand Surg 2002; 27A: 243-251.

80. Johnston Jones K, Sanders WE. Posttraumatic radioulnar instability: treatment by anatomic reconstruction of the volar and dorsal radioulnar ligaments. Orthop Trans 19951996; 19: 832.

81. Darrach W. Forward dislocation of the inferior radioulnar joint, with fracture of the lower third of the shaft of the radius. Ann Surg 1912; 56: 801.

82. Darrach W. Partial excision of the lower shaft of the ulna for deformity following Colles' fracture. Ann Surg 1913; 57: 764.

83. Bowers WH. Distal radioulnar joint arthroplasty: The hemi-resection interposition technique. J Hand Surg (Am) 1985; 10: 169.

84. Scheker LR, Severo A. Ulnar shortening for the treatment of early post-traumatic osteoarthritis at the distal radioulnar joint. J Hand Surg [Br]. 2001 Feb;26(1):41-4.

85. Hartz CR, Beckenbaugh RD. Long-term results of resection of the distal ulna for post-traumatic conditions. J Trauma 1979; 19: 219-26.

86. Dingman VC. Resection of the distal end of the ulna (Darrach Operation): An end-result study of 24 cases. J Bone Joint Surg 1952; 34A: 893-900.

87. Bieber EJ, Linscheid RI, Dobyns JH, et al. Failed distal ulna resections. J Hand Surg 1988; 13A: 193-200.

88. Bell MJ, Hill RJ, McMurtry RY. Ulnar impingement syndrome. J Bone Joint Surg 1985; 67B: 126-9.

89. Gainor BJ, Schaberg J. The rheumatoid wrist after resection of the distal end of the ulna. J Hand Surg (Am) 10: 837844, 1985. 
90. Sauve L, Kapandji M. Nouvelle technique de traitment chirurgical des luxations recidivantes isolees de l'extremite inferieure du cubitus. J Chir ( $\mathrm{Pa}-$ ris) 47: 589-594, 1936.

91. Sanders RA, Frederick HA, Hontas RB. The Sauve-Kapandji procedure: A salvage operation for the distal radioulnar joint. J Hand Surg 1991; 16A: 1125-9.

92. Mikkelsen SS, Lindblad BE, Larsen ER et al. Sauve-Kapandji operation for disorders of the distal radioulnar joint after Colles' fracture. Acta Orthop Scand 68: 64-66, 1997.

93. Vincent KA, Szabo RM, Agee JM. The Sauve-Kapandji for reconstruction of the rheumatoid distal radioulnar joint. $J$ Hand Surg (Am) 18: 978-983; 1993.

94. Rothwell AG, O'Neill L, Cragg K. Sauve-Kapandji procedure for disorders of the distal radioulnar joint: A simplified techqniue. J Hand Surg 1996; 21: 771-777.

95. Lichtman DM, Ganocy K, Kim DC. The indications for and techniques and outcomes of ablative procedures of the distal ulna. Hand Clinics 1998; 14 : 265-77.

96. Nakamura R, Tsunoda K, Watanabe $\mathrm{E}$, et al. The Sauve-Kapandji procedure for chronic dislocation of the distal radioulnar joint with destruction of the articular surface. J Hand Surg (Br) 127-132; 1992.

97. Taleisnik J. The Sauve-Kapandji procedure. Clin Orthop 275: 110-123, 1992.

98. Bowers WH. Distal radioulnar joint arthroplasty: The hemiresection-interposition technique. J Hand Surg (Am) 10: 169178; 1985.

99. Watson HK, Ryu J, Burgess R. Matched distal ulnar resection. J Hand Surg (Am) 11: 812-817, 1986.

100. Bain GI, Pugh DMW, Mac Dermid JC, et al. Matched hemiresection interposition arthroplasty of the distal radioulnar joint. J Hand Surg 1995; 20A: 944-950.

101. Lanz U, Markulin M. Hemiresection interposition arthroplasty of the distal radioulnar joint. In Vastamaki M (ed):Current trends in Hand Surgery. Elsevier Science BV, Amsterdam, Netherlands, 1995, pp 207-212.

102. Minami A, Kaneda K, Itoga $\mathrm{H}$. Hemiresection-interposition arthroplasty of the distal radioulnar joint associated with repair of the triangular fibrocartilage complex lesions. J Hand Surg 1991; 16: 1120-5.

103. Lamey DM, Fernandez DL. Results of the modified SauveKapandji procedure in the treatment of chronic posttraumatic derangement of the distal radioulnar joint. $J$ Bone Joint Surg 1998; 80: 1758-69.

104. Watson HK, Gabuzda GM. Matched distal ulna resection for posttraumatic disorders of the distal radioulnar joint. $J$ Hand Surg 1992; 17(A): 724-30.

105. Swanson AB. Implant arthroplasty for disabilities of the distal radioulnar joint. Ortho Clin 1973; 4: 373.

106. Stanley D, Herbert TJ. The Swanson ulnar head prosthesis for post-traumatic disorders of the distal radio-ulnar joint. J Hand Surg (Br). 1992; 17: $682-8$

107. van Schoonhoven J, Fernandez DL, Bowers WH, Herbert TJ. Salvage of the failed resection arthroplasties of the distal radioulnar joint using a new ulnar head prosthesis. J Hand Surg 2000; 25A: 438-46.

108. Sauerbier M, Hahn ME, Fujita $M$, Neale PG, Berglund LJ, Berger RA. Analysis of dynamic distal radioulnar convergence after ulnar head resection and endoprosthesis implantation. J Hand Surg (Am) 2002; 27: 425-34.

109. Scheker LR. Distal radioulnar joint prostheses to rescue the so-called salvage procedures. In Simmen BR, Allieu Y, Lluch
A, et al (eds): Hand Arthroplasties. London, Martin Dunitz, 2000, p 151.

110. Scheker LR, Ozer K. A simple method of examination for the diagnosis of distal radioulnar joint incongruity. European Journal of Plast Surg, 2004; 27 : 42-43.

111. Lees VC, Scheker LR. The radiological demonstration of dynamic ulnar impingement. $J$ Hand Surg. 1996;22B:448-450.

112. Gummesson C, Atroshi I, Ekdahl C. The disabilities of the arm, shoulder and hand (DASH) outcome questionnaire: longitudinal construct validity and measuring self-rated health change after surgery. BMC Musculoskelet Disord. 2003 Jun 16;4:11.

113. MacDermid JC. Development of a scale for patient rating of wrist pain and disability. J Hand Therapy 1996;9:178-183.

114. R. Zimmermann, M. Gschwentner, R. Arora, C. Harpf, M. Gabl, and S. Pechlaner. Treatment of distal radioulnar joint disorders with a modified Sauve-Kapandji procedure: long-term outcome with special attention to the DASH Questionnaire. Arch.Orthop.Trauma Surg. 123 (6): 293-298, 2003.

115. Bieber E. J., Linscheid R. L., Dobyns J. H., and Beckenbaugh R. D. Failed distal ulna resections. J.Hand Surg.[Am.] 13 (2):193-200, 1988.

116. Schneider L. H. and Imbriglia J. E. Radioulnar joint fusion for distal radioulnar joint instability. Hand Clin. 7 (2):391-395, 1991.

117. Ragab A.A., Kraay M.J., Goldberg V.M. Clinical and radiographic outcomes of total hip arthroplasty with insertion of an anatomically designed femoral component without cement for the treatment of primary osteoarthritis: A study with a minimum of six years of follow-up. Journal of Bone and Joint Surgery-Series A Volume 81, Issue 2, 1999, Pages $210-218$ 